\title{
MODELOS DE SUSCEPTIBILIDADE A DESLIZAMENTOS SUPERFICIAIS TRANSLACIONAIS NA REGIÃO A NORTE DE LISBOA
}

\author{
Aldina Piedade ${ }^{1}$ \\ José Luís ZÊZERE ${ }^{1}$ \\ RicARdo A. C. GARCIA ${ }^{1}$ \\ SÉrgio C. Oliveira ${ }^{1}$
}

\begin{abstract}
Resumo - Na última década, os Sistemas de Informação Geográfica (SIG) têm permitido a criação e o cruzamento de parâmetros de forma cada vez mais rápida e fiável, justificando o incremento das variáveis de entrada nos modelos de avaliação da susceptibilidade aos movimentos de vertente. Neste contexto, a análise sensitiva aos modelos preditivos surge com o objectivo de aferir a importância relativa dos factores de predisposição, permitindo distinguir entre aqueles que mais contribuem para melhorar a performance do modelo de susceptibilidade e os que pouco acrescentam aos resultados finais. Neste trabalho é efectuada uma análise sensitiva a modelos de susceptibilidade a deslizamentos translacionais superficiais em duas áreas amostra na região a Norte de Lisboa, inseridas no mesmo contexto geológico e geomorfológico: área de Fanhões-Trancão e área de Lousa-Loures. Os resultados obtidos permitiram identificar um conjunto de três variáveis que se destacam pela associação espacial aos deslizamentos translacionais superficiais nas duas áreas estudadas: declive, unidades geomorfológicas e exposição das vertentes. Adicionalmente, demonstra-se que não existe uma relação linear entre o número de variáveis presentes no modelo e a respectiva capacidade preditiva, e mostra-se que é viável a produção de mapas de susceptibilidade consistentes, recorrendo a um pequeno grupo de variáveis que têm uma forte relação espacial com os movimentos de vertente.
\end{abstract}

Palavras-chave: Susceptibilidade, deslizamentos translacionais superficiais, factores de predisposição, análise sensitiva.

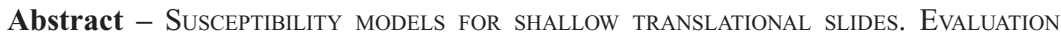
AND MAPPING In the REgion to the North OF Lisbon. Over the last decade, Geographic Information Systems (GIS) have enabled the creation and the rigorous crossing and computation
}

Recebido: Novembro, 2010. Aceite: Fevereiro, 2011.

1 Centro de Estudos Geográficos. Instituto de Geografia e Ordenamento do Território. Universidade de Lisboa.E-mails: aldinapiedade@campus.ul.pt; zezere@campus.ul.pt; rgarcia@campus.ul.pt; cruzdeoliveira@campus.ul.pt 
of cartographic thematic layers. Thus, it has been possible to increase the number of variables within data-driven landslide susceptibility models. In this context, sensitivity analysis is an analytical tool used to assess the relative importance of the landslide predisposing factors into a particular landslide susceptibility model. In this study, a sensitivity analysis of shallow translational slides susceptibility models is made for two test sites in the Region to the North of Lisbon, which have the same geologic and geomorphologic context: the Fanhões-Trancão test site and the Lousa-Loures test site. The results obtained allowed for the identification of 3 variables that have the highest spatial association with shallow translational slides in both test sites: slope angle, geomorphology and slope aspect. Furthermore, results prove the absence of a linear relationship between the number of variables within a landslide predictive model and the model prediction capacity. In addition to this, it is shown that reliable landside susceptibility maps can be produced based on a small group of variables that have a strong spatial relationship with slope movements.

Key words: Susceptibility, shallow translational slides, predisposing factors, sensitivity analysis.

Résumé - ModĖLes De SUSCEPTIBILITÉ AUX GLISSEMENTS PLANS SUPERFICIELS DANS LA RÉGION AU NoRd DE LisbonNe. Lors de la dernière décennie, les Systèmes d'Information Géographique (SIG) ont permis de créer et de croiser des paramètres avec de plus en plus de fiabilité et de rapidité. Ainsi, on a pu augmenter le nombre de variables d'entrée dans les modèles d'évaluation de la susceptibilité aux mouvements de terrain. L'analyse sensitive est un outil analytique utilisé pour évaluer l'importance relative des facteurs de prédisposition aux mouvements de terrain dans un modèle de susceptibilité particulier, permettant ainsi de distinguer les facteurs qui contribuent à l'amélioration de la performance du modèle de ceux qui influencent peu les résultats finaux. Dans ce travail, une analyse sensitive des modèles de susceptibilité aux glissements plans superficiels est effectuée et appliquée à deux zones d'études de la région au nord de Lisbonne et qui s'inscrivent dans le même contexte géologique et géomorphologique : la zone de Fanhões-Trancão et celle de Lousa-Loures. Les résultats obtenus permettent d'identifier un ensemble de trois variables qui se distinguent par une forte association spatiale avec les glissements plans superficiels des deux zones d'étude : les unités géomorphologiques et l'inclinaison et l'exposition des pentes. De plus, les résultats prouvent l'absence de relation linéaire entre le nombre de variables présentes dans le modèle et sa capacité prédictive. Enfin, on montre que la production de cartes de susceptibilité est viable lorsqu'elle est basée sur un petit groupe de variables ayant une forte relation spatiale avec les mouvements de terrain.

Mots clés: Susceptibilité, glissements plans superficiels, facteurs de prédisposition, analyse sensitive

\section{INTRODUÇÃO}

Para um ordenamento do território eficaz e uma gestão equilibrada do risco, é necessário conhecer a instabilidade geomorfológica presente e passada e a predisposição do território para a ocorrência de movimentos de vertente (Zêzere, 2007). Este conhecimento implica a avaliação da susceptibilidade, entendida por Soeters e Van Westen (1996) como a propensão do território para a ocorrência de um tipo particu- 
lar de movimento de vertente em tempo indeterminado, com base num conjunto de factores de predisposição à instabilidade geomorfológica, não contemplando a probabilidade de ocorrência ou o período de retorno.

No contexto da avaliação da susceptibilidade, é assumido que os futuros movimentos de vertente deverão ocorrer sob as mesmas condições que determinaram a instabilidade no passado (Varnes, 1984; Carrara et al., 1999). Esta assunção deriva da aplicação prospectiva do principio do uniformitarismo ( $O$ passado e o presente são as chaves para o futuro), no pressuposto de que as mesmas causas são susceptíveis de gerar os mesmos efeitos (Varnes, 1984; Van Westen et al., 2008).

A avaliação da susceptibilidade é dominada, actualmente, pelo recurso a métodos de cartografia indirecta, de onde se destacam os de base estatística (Guzzetti et al., 2000; Chacón et al., 2006). Com a utilização destes métodos a ponderação dos factores que condicionam a instabilidade é obtida através da sua representação cartográfica e da análise das suas relações espaciais com a distribuição dos movimentos de vertente. Adicionalmente, estes métodos permitem reduzir a subjectividade na determinação da susceptibilidade, uma vez que as técnicas de aquisição, processamento, análise e representação da informação são normalizadas (Carrara, 1993).

Na última década, os Sistemas de Informação Geográfica (SIG) têm permitido a criação e o cruzamento de parâmetros de forma cada vez mais rápida e fiável, justificando o incremento das variáveis de entrada nos modelos de avaliação da susceptibilidade. Van Westen et al. (2008) listam um total de 29 variáveis com eventual relevância no sistema da instabilidade de vertentes, agrupadas em seis temas: morfologia/morfometria, geologia, solos, hidrologia, geomorfologia e uso do solo. Em trabalhos recentes dedicados à avaliação da susceptibilidade de ocorrência de movimentos de vertente suportados por SIG (e.g. Remondo et al., 2003; Santacana et al., 2003; Lee, 2004; Van Den Eeckhaut et al., 2010) é recorrente a utilização de mais de uma dezena de variáveis assumidas como factores de predisposição para a instabilidade geomorfológica.

Pese embora a disponibilidade actual de ferramentas analíticas, que possibilitam o manuseamento de grande quantidade de informação em simultâneo, tem sido pouco explorada a avaliação do peso individual dos factores de predisposição à instabilidade geomorfológica, assim como a determinação dos incrementos preditivos que resultam da inclusão de mais variáveis nos modelos de susceptibilidade.

Este trabalho tem como objectivo a realização de uma análise sensitiva (Remondo et al., 2003; Zêzere et al., 2005a) aos factores de predisposição utilizados na construção de modelos de susceptibilidade a deslizamentos translacionais superficiais, em duas áreas amostra da região a Norte de Lisboa, inseridas no mesmo contexto geológico e geomorfológico: Fanhões-Trancão e Lousa-Loures. Estas áreas são acompanhadas desde meados da década de 1980, o que permitiu a realização prévia de modelos de susceptibilidade geomorfológica devidamente validados (Zêzere et al., 2004; Piedade, 2009; Piedade et al., 2010a).

Os modelos anteriormente obtidos basearam-se em inventários de movimentos de vertente e num conjunto de 7 factores de predisposição, idênticos para ambas as 
áreas. Neste contexto, importa perceber se o aumento da quantidade de informação relativa aos factores de predisposição gera modelos de susceptibilidade com melhor capacidade preditiva. Assim, a análise sensitiva é aplicada no sentido de perceber como se comportam os diferentes factores de predisposição, quando utilizados como variáveis independentes num modelo de susceptibilidade a deslizamentos translacionais superficiais, tentando perceber quais os que mais contribuem para a explicação da distribuição espacial dos movimentos, identificando os factores de predisposição mais eficazes para a discriminação das áreas instáveis e estáveis, no que se refere ao tipo de deslizamento considerado.

O desenvolvimento do trabalho em duas áreas com contexto geológico e geomorfológico similar é feito com o objectivo de atribuir uma maior robustez ao exercício da análise sensitiva e aos próprios resultados dos modelos de susceptibilidade, aferindo se os factores de predisposição que mais contribuem para a boa resolução dos modelos são os mesmos em ambas as áreas.

\section{II. ÁREA DE ESTUDO, INSTABILIDADE DE VERTENTES E FACTORES DE PREDISPOSIÇÃO}

As áreas de Fanhões-Trancão e de Lousa-Loures, com uma superfície de $20 \mathrm{~km}^{2}$ e $17 \mathrm{~km}^{2}$, respectivamente, inserem-se na região a Norte de Lisboa, localizando-se no concelho de Loures. Estas duas áreas amostra são parte integrante da costeira Lousa-Bucelas, sendo marcadas por uma estrutura monoclinal com inclinações de $12^{\circ}$ para S no caso de Fanhões-Trancão e entre $8^{\circ}$ a $30^{\circ}$ para SSE a SE em Lousa-Loures (Ferreira, 1984; Zêzere, 1997). Do ponto de vista litológico observa-se alternância de terrenos com diferente resistência mecânica, permeabilidade, alterabilidade e resistência ao corte. Destacam-se, pela extensão dos afloramentos, as rochas do complexo vulcânico de Lisboa e as rochas sedimentares de idade cretácica como os calcários, arenitos, calcários margosos, pelitos e dolomitos (Zbyszewski, 1964, Zêzere, et al., 1999, 2005a; Piedade et al., 2010a) como se vê na fig. 1 .

A base de dados dos deslizamentos translacionais superficiais consiste num inventário, obtido através de trabalho de campo para ambas as áreas, efectuado por Zêzere (1997) registada no quadro I. Os deslizamentos translacionais superficiais são movimentos de vertente com superfície de ruptura planar, que se desenvolvem paralelamente à superfície topográfica, a uma profundidade típica inferior a $1,5 \mathrm{~m}$. O plano de ruptura ocorre, frequentemente, no contacto entre depósitos de vertente permeáveis e o substrato rochoso impermeável (Zêzere et al., 2005b).

$\mathrm{Na}$ área de Fanhões-Trancão foram inventariados 100 deslizamentos translacionais superficiais (densidade de 5 movimentos $/ \mathrm{km}^{2}$ ), a que correspondem $142172 \mathrm{~m}^{2}$ de área instabilizada $(0,71 \%$ da área total). Para a área de Lousa-Loures inventariaram-se 82 deslizamentos do mesmo tipo (densidade de 4,8 movimentos $/ \mathrm{km}^{2}$ ), correspondentes a uma área instabilizada de $37099 \mathrm{~m}^{2}$ (0,22\% da área total). 

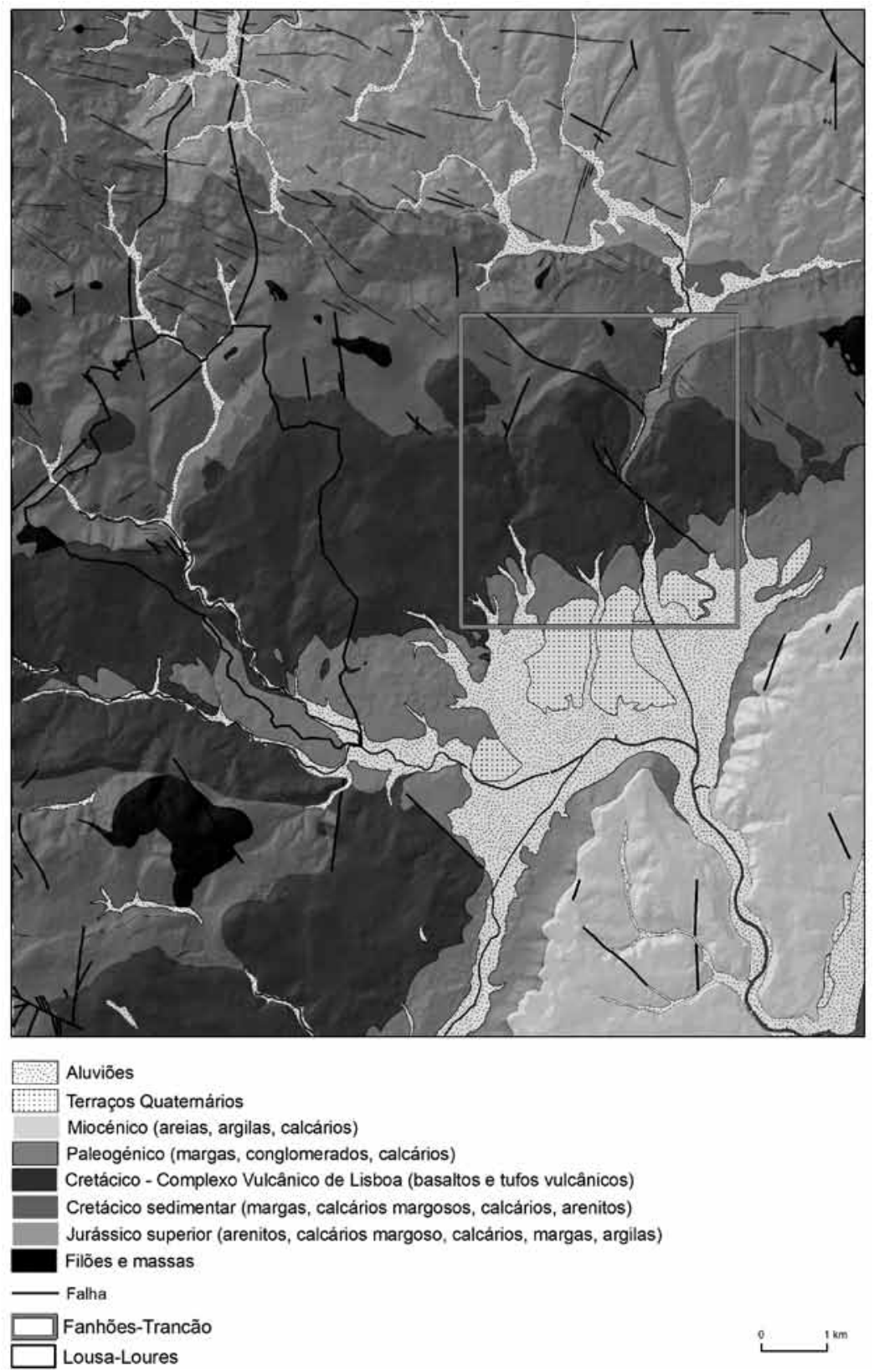

Fig. 1 - Geologia da Região a Norte de Lisboa e localização das áreas de Lousa-Loures e de Fanhões-Trancão.

Fig. 1 - Geologic map of the Region to the North of Lisbon and localization of the Lousa-Loures and Fanhões-Trancão test sites. 
Quadro I - Inventário de deslizamentos translacionais superficiais nas áreas de estudo. Table I - Inventory of shallow translational slides in test sites.

\begin{tabular}{ccccc}
\hline & $\begin{array}{c}\mathrm{N}^{\circ} \text { de } \\
\text { eventos }\end{array}$ & $\begin{array}{c}\text { Área } \\
\text { instabilizada }\left(\mathrm{m}^{2}\right)\end{array}$ & $\begin{array}{c}\text { Densidade de } \\
\text { movimentos }\left(\mathrm{n} / \mathrm{km}^{2}\right)\end{array}$ & $\begin{array}{c}\text { Total de área } \\
\text { instabilizada (\%) }\end{array}$ \\
\hline Fanhões-Trancão & 100 & 142172 & 5,0 & 0,71 \\
Lousa-Loures & 82 & 37099 & 4,8 & 0,22 \\
\hline
\end{tabular}

Os factores de predisposição da instabilidade geomorfológica, assumidos como capazes de predizer a distribuição espacial dos deslizamentos e usados na construção dos modelos de susceptibilidade para as duas áreas, são os seguintes (quadro II): declive ( 8 classes), exposição das vertentes ( 9 classes), perfil transversal das vertentes (5 classes), unidades litológicas (6 classes), depósitos superficiais (7 classes), unidades geomorfológicas (12 classes) e uso do solo (6 classes). No total observam-se 52 e 48 classes, nas áreas de Fanhões-Trancão e de Lousa-Loures, respectivamente, valores que se justificam pela ausência pontual de algumas classes numa das áreas. O quadro II sintetiza ainda as fontes de informação e os procedimentos utilizados para a construção de cada tema independente. Destaca-se a vectorização e edição de informação de cartografia pré-existente, em formato analógico e digital, a interpretação de ortofotomapas e uma forte componente de trabalho de campo.

Quadro II - Variáveis e classes consideradas como factores de predisposição da instabilidade das vertentes.

Table II - Predisposing factor classes of slope instability.

\begin{tabular}{|c|c|c|c|c|}
\hline Classes & Código & $\begin{array}{c}\text { Fanhões-Trancão } \\
\left(n .^{\circ} \text { de pixels }\right)\end{array}$ & $\begin{array}{l}\text { Lousa-Loures } \\
\text { (n. }{ }^{\circ} \text { de pixels) }\end{array}$ & $\begin{array}{c}\text { Fontes de } \\
\text { Informação }\end{array}$ \\
\hline $0-5$ & D1 & 226885 & 133541 & \multirow{8}{*}{$\begin{array}{c}\text { Modelo Digital do } \\
\text { Terreno } \\
(\text { Pixel }=5 \mathrm{~m})\end{array}$} \\
\hline ]5-10] & $\mathrm{D} 2$ & 265609 & 229100 & \\
\hline ]10-15] & D3 & 148501 & 136991 & \\
\hline ]15-20] & D4 & 71497 & 75723 & \\
\hline ]20-25] & D5 & 29093 & 46509 & \\
\hline ]25-30] & D6 & 27128 & 28618 & \\
\hline ]30-40] & D7 & 23121 & 21810 & \\
\hline$>40$ & D8 & 6275 & 2822 & \\
\hline Terreno Plano & EV1 & 1111 & 13548 & \multirow{9}{*}{$\begin{array}{c}\text { Modelo Digital do } \\
\text { Terreno } \\
\text { (Pixel }=5 \mathrm{~m})\end{array}$} \\
\hline $\mathrm{N}$ & EV2 & 28818 & 37770 & \\
\hline $\mathrm{NE}$ & EV3 & 61879 & 74355 & \\
\hline E & EV4 & 111803 & 86653 & \\
\hline SE & EV5 & 151278 & 112632 & \\
\hline S & EV6 & 206579 & 117393 & \\
\hline SW & EV7 & 104967 & 110453 & \\
\hline W & EV8 & 78902 & 81176 & \\
\hline NW & EV9 & 42732 & 41134 & \\
\hline
\end{tabular}




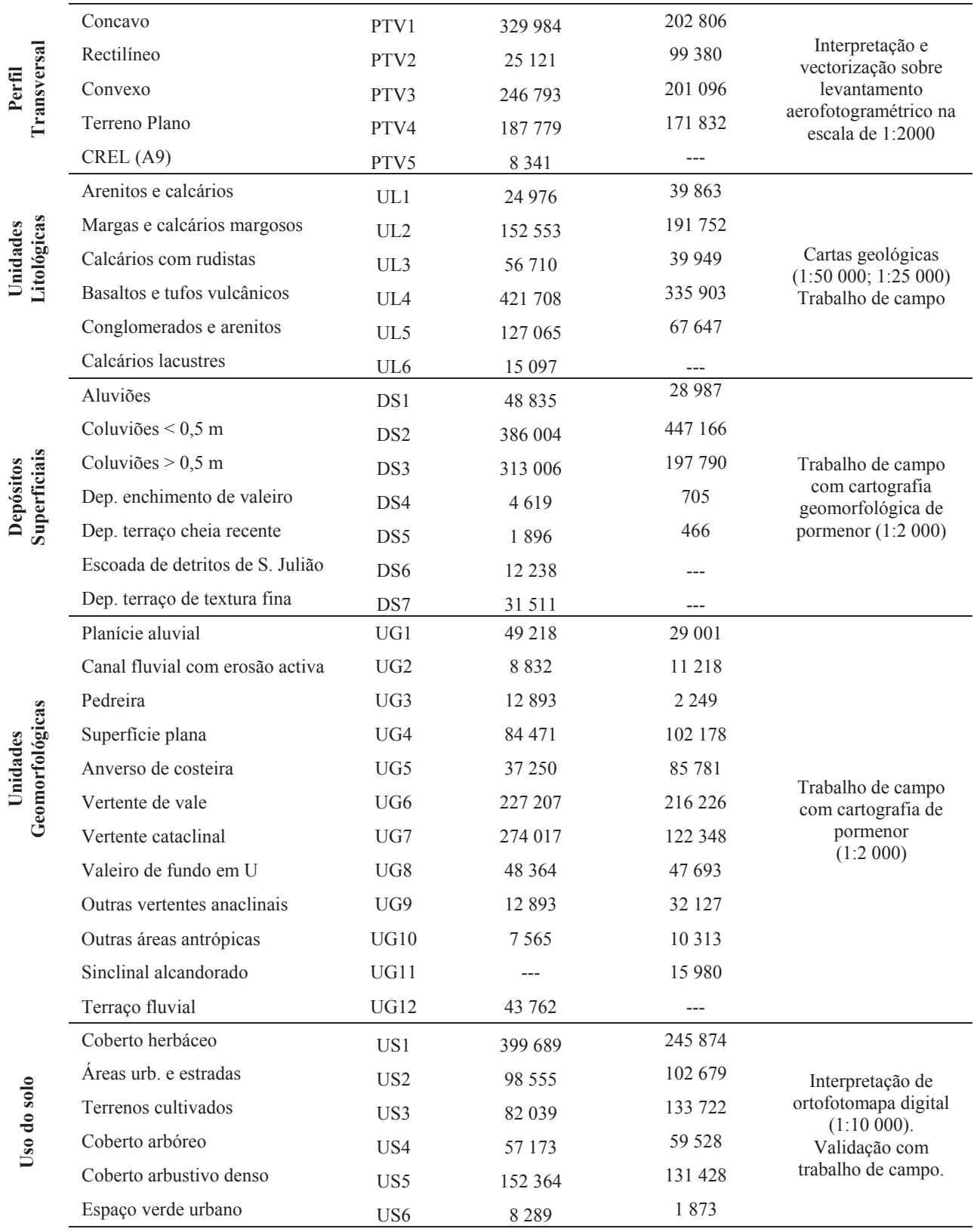

A similitude das áreas amostra de Trancão-Fanhões e Lousa-Loures é demonstrada pela concordância geral das frequências absolutas das diferentes classes nas duas áreas, expressas pelos respectivos números de pixels (quadro II). 
Para a utilização das variáveis assumidas como factores de predisposição nos modelos de susceptibilidade, foram criadas estruturas de dados matriciais com um pixel de $5 \mathrm{~m}$ (células de $25 \mathrm{~m}^{2}$ ). Assim, para a área de Fanhões-Trancão cada variável é formada por um raster de 798109 pixels, enquanto para a área de Lousa-Loures esse valor é de 675114 pixels

\section{METODOLOGIA}

O primeiro passo no processo de integração cartográfica dos dados é o cálculo dos scores de susceptibilidade para cada classe de variável, para ambas as áreas amostra. $\mathrm{O}$ método estatístico bivariado utilizado, para a avaliação da susceptilibidade à ocorrência de movimentos de vertente nas áreas de Fanhões-Trancão e Lousa-Loures, foi o do Valor Informativo (Yan, 1988; Yin e Yan, 1988; Piedade, 2009; Piedade et al., 2010b). Esta técnica descreve quantitativamente, sob a forma de scores, as relações existentes entre cada classe de cada tema e os movimentos de vertente (Yin e Yan, 1988). O método do Valor Informativo tem uma base Bayesiana, sustentando-se na transformação logarítmica (log natural) da razão entre probabilidade condicionada e probabilidade à priori. Com este método é possível ponderar cada classe de cada factor predisposição da instabilidade de vertentes, de forma objectiva, quantificada e reprodutível.

O Valor Informativo (Ii) para qualquer variável independente $X i$ foi determinado pela seguinte equação:

$$
I i=\log \frac{S i / N i}{S / N}
$$

Onde:

$S i=$ n. $^{\circ}$ de pixels com deslizamentos translacionais superficiais na variável $X i$;

$N i=\mathrm{n} .{ }^{\circ}$ de pixels com a variável $X i$;

$S=\mathrm{n}^{\circ}{ }^{\circ}$ total de pixels com deslizamentos translacionais superficiais na área amostra;

$N=\mathrm{n} .{ }^{\circ}$ total de pixels na área amostra.

Devido à normalização logarítmica, $I i$ não é determinável quando $S i=0$. Nestes casos, o valor de $I i$ foi assumido como igual ao $I i$ mais baixo determinado para o conjunto das variáveis, em cada uma das áreas amostra.

Valores positivos de $I i$ indicam uma relação positiva entre presença da classe da variável e ocorrência de movimentos de vertente, tanto mais elevada quanto maior o valor do score. Valores negativos de Ii denunciam que a distribuição dos movimentos de vertente é pouco controlada pela classe da variável em questão. 
O valor de susceptibilidade para cada pixel $j$ foi calculado pelo Valor Informativo total dado pela seguinte equação:

$$
I j=\sum_{i=1}^{n} X j i . I i
$$

Onde:

$n=$ n. $^{\circ}$ de variáveis;

$X i j$ é igual a 1 ou 0 , consoante a variável $X i$ está ou não presente no pixel $j$, respectivamente.

Como foi anteriormente referido, a análise sensitiva foi efectuada com o objectivo de determinar a importância relativa de cada factor de predisposição na capacidade preditiva dos modelos de susceptibilidade. Neste trabalho pretende-se clarificar, nas duas áreas amostra estudadas, quais as variáveis que têm mais capacidade explicativa e com quantas variáveis se obtém a melhor taxa de validação. Neste contexto, existem dois conjuntos de informação que estiveram presentes na primeira fase da análise sensitiva: (i) o inventário dos movimentos de vertente; e (ii) os factores de predisposição considerados isoladamente com as respectivas classes. Assim, cada factor de predisposição foi cruzado autonomamente com o conjunto de movimentos de vertente da própria área, gerando um modelo de susceptibilidade simples.

A validação de cada modelo de susceptibilidade, sustentado por um único factor de predisposição, foi efectuada com recurso à construção de uma curva de sucesso (Fabbri et al., 2002; Chung e Fabbri, 2005). Os resultados obtidos foram ordenados hierarquicamente, permitindo comparar a importância relativa de cada factor.

A hierarquia atrás referida foi respeitada na segunda fase da análise sensitiva, que passou pela introdução sucessiva de uma nova variável no modelo de susceptibilidade, a cada passo da análise (e.g. $\mathrm{M}_{2}=\mathrm{f}\left(\mathrm{V}_{1}+\mathrm{V}_{2}\right) ; \mathrm{M}_{3}=\mathrm{f}\left(\mathrm{V}_{1}+\mathrm{V}_{2}+\mathrm{V}_{3}\right) ; \mathrm{M}_{\mathrm{n}}=\mathrm{f}$ $\left.\left(\mathrm{V}_{1}+\mathrm{V}_{2}+\mathrm{V}_{3}+\mathrm{V}_{4}+\ldots \mathrm{V}_{\mathrm{n}}\right)\right)$. Para cada modelo obtido $\left(\mathrm{M}_{2} \ldots \mathrm{M}_{\mathrm{n}}\right)$ foi calculada a Ârea Abaixo da Curva (AAC), com o objectivo de perceber qual a combinação de variáveis que obtém melhor capacidade preditiva (Zêzere et al., 2005a, 2008; Guzzetti et al., 2006; Piedade, 2009). Este exercício foi efectuado, de modo autónomo, para as duas áreas amostra estudadas.

\section{RESULTADOS E DISCUSSÃO}

A figura 2 representa os Valores Informativos obtidos para cada classe de variável nas áreas amostra de Fanhões-Trancão e de Lousa-Loures. Em regra, as classes de variável que mais contribuem para a explicação dos deslizamentos translacionais superficiais numa área são as mesmas que mais controlam os movimentos de vertente na outra área. Adicionalmente, as variáveis que apresentam Valor Informativo negativo tendem também a repetir-se nas duas áreas, o que indica que as condições de terreno que melhor definem a estabilidade do território são idênticas nas duas áreas amostra. 

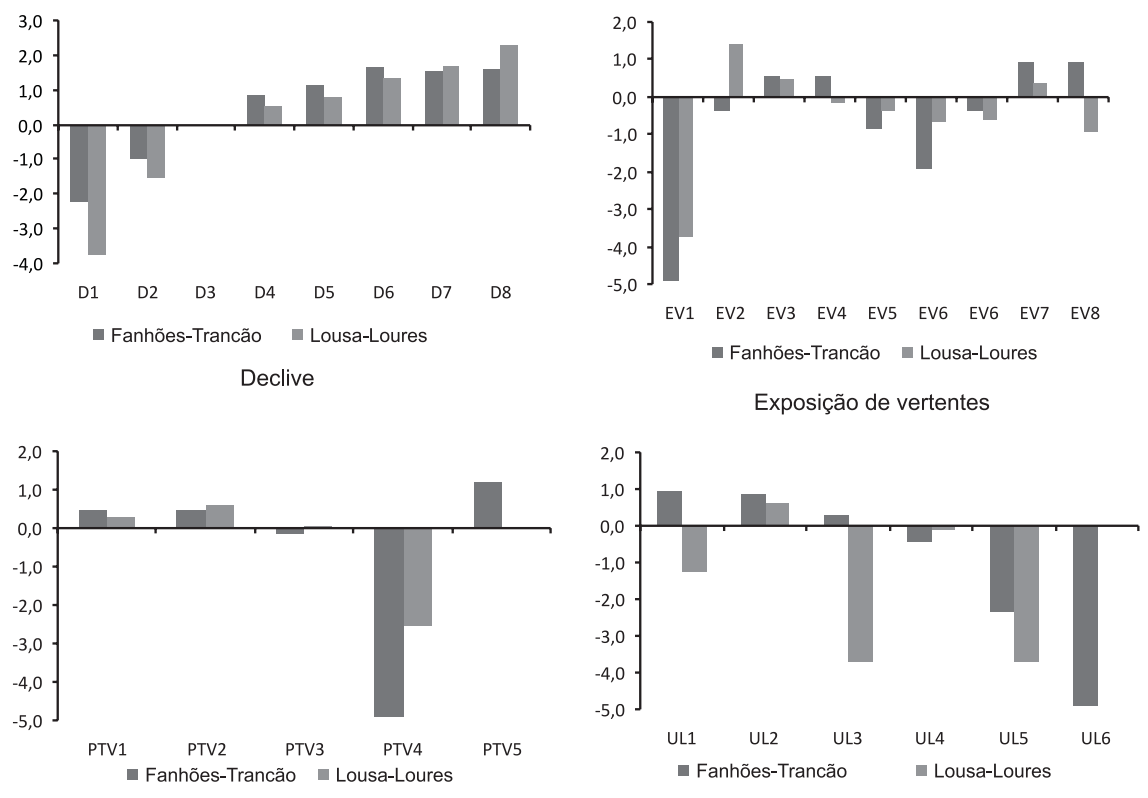

Perfil transversal das vertentes

Unidades litológicas
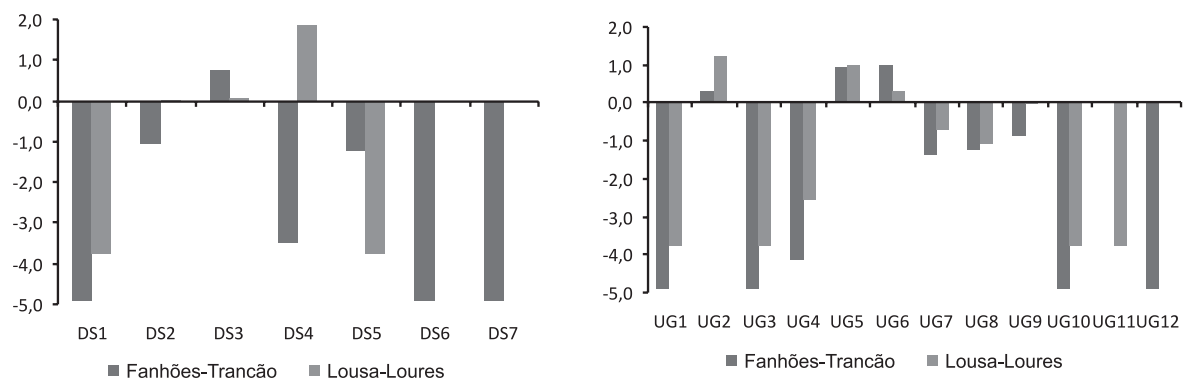

Depósitos superficiais

- Fanhões-Trancão — Lousa-Loures

Unidades geomorfológicas

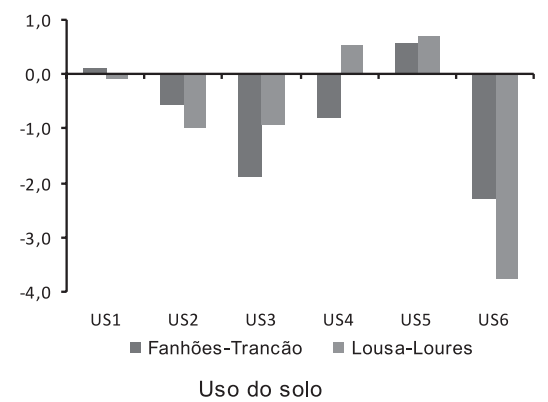

Fig. 2 - Valores Informativos das classes dos factores de predisposição nas áreas amostra de Lousa-Loures e Fanhões-Trancão.

Fig. 2 - Information Value of predisposing factor classes in the Lousa-Loures and Fanhões-Trancão test sites. 
Pela leitura da figura 2 é possível concluir que a ocorrência de deslizamentos translacionais superficiais nas áreas de Fanhões-Trancão e de Lousa-Loures é favorecida em vertentes com declives superiores a $25^{\circ}$ (D6, D7 e D8), expostas a N (EV2) ou a NW e W (EV9 e EV8), talhadas em margas e calcários margosos (UL2), em contexto de anverso de costeira (UG5). A presença de canais fluviais marcados por erosão activa (UG2) e de vertentes de vale (UG6) são igualmente condições de predisposição relevantes para a instabilidade geomorfológica.

O quadro III apresenta a hierarquia dos factores de predisposição que contribuem para a explicação dos deslizamentos translacionais superficiais na área de Fanhões-Trancão. Esta hierarquia foi definida de acordo com a AAC da curva de sucesso dos modelos de susceptibilidade produzidos com cada variável isoladamente. Através desde exercício, verificou-se que, como seria de esperar, o declive foi o parâmetro que mais contribuiu para a explicação deste tipo de movimento de vertentes, com uma AAC de 0,802 . No top 3 das variáveis mais explicativas, para além do declive, encontraram-se as unidades geomorfológicas e a exposição das vertentes. No final desta hierarquia, com menor capacidade de discriminação entre áreas deslizadas e não deslizadas, surge o uso do solo, com 0,631 de AAC.

Quadro III - Hierarquia dos factores de predisposição para os deslizamentos translacionais superficiais na área de Fanhões-Trancão.

Table III - Ranking of predisposing factors of shallow translational slides

in the Fanhões-Trancão test site.

\begin{tabular}{clc}
\hline Hierarquia & Variável & AAC \\
\hline 1 & Declive & 0,802 \\
2 & Unidades geomorfológicas & 0,788 \\
3 & Exposição das vertentes & 0,738 \\
4 & Depósitos superficiais & 0,731 \\
5 & Unidades litológicas & 0,706 \\
6 & Perfil transversal das vertentes & 0,672 \\
7 & Uso do solo & 0,631 \\
\hline
\end{tabular}

O quadro IV sistematiza a hierarquia dos factores de predisposição que contribuem para a explicação dos deslizamentos translacionais superficiais em Lousa-Loures. À semelhança do observado na área de Fanhões-Trancão, verifica-se que o declive é a variável que mais contribui para a explicação do tipo de movimentos de vertente considerado, com uma AAC de 0,806. No top 3 das variáveis mais explicativas, para além do declive, encontram-se, novamente, as unidades geomorfológicas e a exposição das vertentes. No final da hierarquia surgem os depósitos superficiais com 0,526 de AAC.

A hierarquia estabelecida nos quadros III e IV foi respeitada para o posterior desenvolvimento de novos modelos de susceptibilidade, para ambas áreas amostra, com a introdução de uma nova variável em cada passo. Os quadros V e VI e as figuras 3 e 4 representam, respectivamente, as AAC e as curvas de sucesso dos resultados obtidos, para as duas áreas amostra. 
Quadro IV - Hierarquia dos factores de predisposição para os deslizamentos translacionais superficiais na área de Lousa-Loures.

Table IV - Ranking of predisposing factors of shallow translational slides in the Lousa-Loures test site

\begin{tabular}{cll}
\hline Hierarquia & Variável & AAC \\
\hline 1 & Declive & 0,806 \\
2 & Unidades geomorfológicas & 0,739 \\
3 & Exposição das vertentes & 0,682 \\
4 & Unidades litológicas & 0,661 \\
5 & Uso do solo & 0,661 \\
6 & Perfil transversal das vertentes & 0,656 \\
7 & Depósitos superficiais & 0,526 \\
\hline
\end{tabular}

Quadro V - AAC de modelos de susceptibilidade a deslizamentos translacionais superficiais na área de Fanhões-Trancão com 2 a 7 factores de predisposição.

Table V-AUC of shallow translational slides susceptibility models in the Fanhões-Trancão test site using 2 to 7 predisposing factors.

\begin{tabular}{lc}
\hline \multicolumn{1}{c}{ Variável } & AAC \\
\hline 2 Variáveis (D+UG) & 0,802 \\
3 Variáveis (D+UG+EV) & 0,843 \\
4 Variáveis (D+UG+EV+DS) & 0,858 \\
5 Variáveis (D+UG+EV+DS+UL) & 0,868 \\
6 Variáveis (D+UG+EV+DS+UL+PTV) & 0,874 \\
7 Variáveis (D+UG+EV+DS+UL+PTV+US) & 0,878 \\
\hline
\end{tabular}

Quadro VI - AAC de modelos de susceptibilidade aos deslizamentos translacionais superficiais na área de Lousa-Loures com 2 a 7 factores de predisposição.

Table VI - AUC of shallow translational slide susceptibility models in the Lousa-Loures test site using 2 to 7 predisposing factors.

\begin{tabular}{ll}
\hline Hierarquia $\quad$ Variável & AAC \\
\hline 2 Variáveis (D+UG) & 0,818 \\
3 Variáveis (D+UG+EV) & 0,825 \\
4 Variáveis (D+UG+EV+UL) & 0,819 \\
5 Variáveis (D+UG+EV+UL+US) & 0,840 \\
6 Variáveis (D+UG+EV+UL+US+PTV) & 0,838 \\
7 Variáveis (D+UG+EV+UL+US+PTV+DS) & 0,839 \\
\hline
\end{tabular}




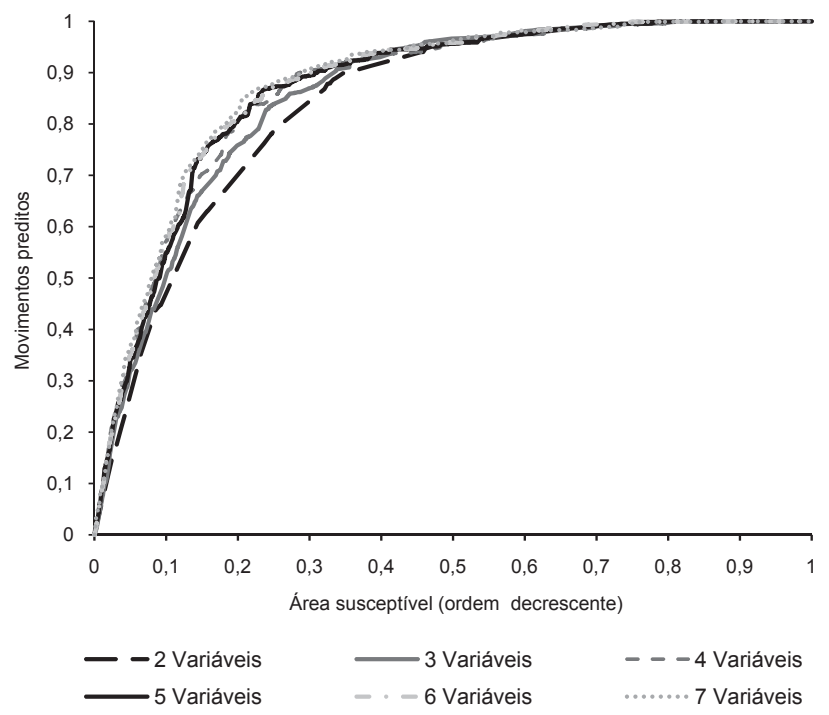

Fig. 3 - Curvas de sucesso dos modelos de susceptibilidade para deslizamentos superficiais translacionais na área de Fanhões-Trancão.

Fig. 3 - Success rate curves of shallow translational slide susceptibility models in the Fanhões-Trancão test site.

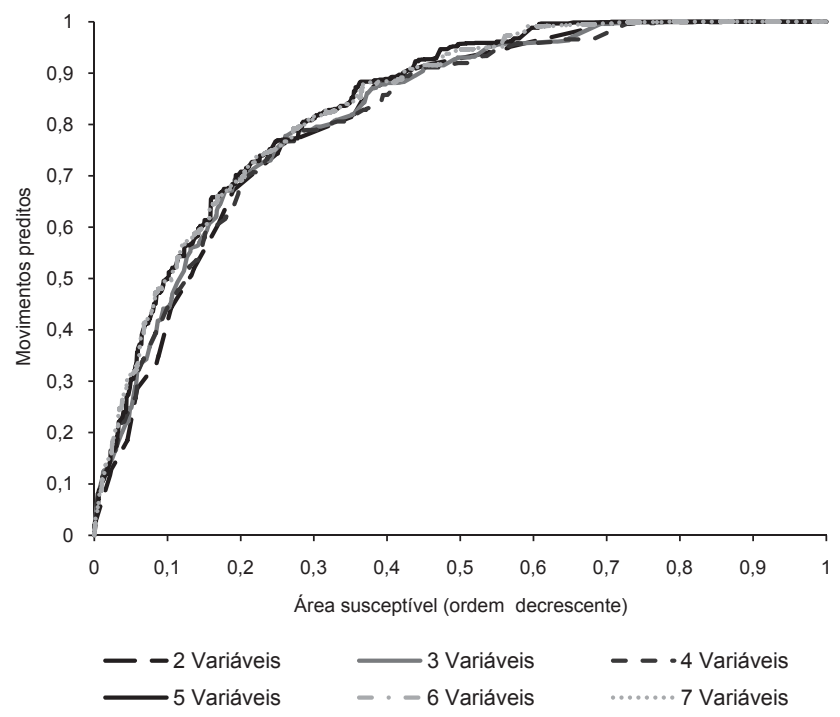

Fig. 4 - Curvas de sucesso dos modelos de susceptibilidade para deslizamentos superficiais translacionais na área de Lousa-Loures.

Fig. 4 - Success rate curves of shallow translational slide susceptibility models in the Lousa-Loures test site. 
Os modelos de susceptibilidade obtidos com diferentes combinações de variáveis nas áreas amostra de Fanhões-Trancão e Lousa-Loures apresentam uma capacidade preditiva bastante idêntica e muito satisfatória (curvas de sucesso com AAC sempre acima de 0,8 ). Analisando os resultados, sistematizados nos quadros V e VI, pode concluir-se que a modelação da susceptibilidade com 2, 3, 4, 5, 6 ou 7, variáveis não produz diferenças muito significativas ao nível da capacidade preditiva dos modelos, como se demonstra pelas reduzidas diferenças nas AAC representadas graficamente nas figuras 3 e 4 . Com efeito, as diferenças nas performances preditivas dos vários modelos de susceptibilidade, medidas pelas AUC, são de 0,08 na área amostra de Fanhões-Trancão e não vão além de 0,02 na área amostra de Lousa-Loures.

No caso da área amostra de Fanhões-Trancão verifica-se que a introdução de uma variável adicional em cada passo da análise sensitiva acrescenta maior capacidade preditiva aos modelos de susceptibilidade, pelo que a melhor performance é obtida pelo modelo que integra 7 variáveis (quadro V). Contudo, as curvas de sucesso mantêm-se muito próximas (fig. 3), assim como as AAC, nos modelos com 3, 4, 5,6 e 7 variáveis. Na área amostra de Lousa-Loures verifica-se que o incremento de mais variáveis no modelo de susceptibilidade não gera, necessariamente, melhores resultados preditivos (quadro VI). Com efeito, o valor mais alto de AAC não se verifica no modelo que utiliza 7 variáveis $(\mathrm{AAC}=0,839)$, mas antes naquele que integra 5 varáveis $(\mathrm{AAC}=0,840)$. Como no caso anterior, as curvas de sucesso mantêm-se sempre muito próximas (fig. 4), principalmente nos modelos que utilizam de 3 a 7 variáveis.

Deste modo, verifica-se que os modelos de susceptibilidade produzidos com a integração das 3 variáveis que evidenciam uma maior correlação espacial com os deslizamentos translacionais superficiais (declive, unidades geomorfológicas e exposição das vertentes) obtêm resultados muito satisfatórios na predição de ocorrência deste tipo de movimento de vertente nas áreas amostra de Fanhões-Trancão e Lousa-Loures, podendo constituir uma boa solução em termos de custo (esforço de recolha/tratamento/computação de dados) - benefício (qualidade dos resultados).

As figuras 5 e 6 apresentam os mapas de susceptibilidade obtidos para as áreas de Fanhões-Trancão (fig. 5) e de Lousa-Loures (fig. 6), utilizando o conjunto de 7 variáveis (fig. 5A e 6A) e as 3 variáveis atrás referidas (fig. 5B e 6B). A expressão cartográfica dos resultados obtidos mostra que, para além da similitude das AAC e das curvas de sucesso, verifica-se uma concordância espacial assinalável nos mapas de susceptibilidade produzidos com 3 e 7 variáveis em qualquer das áreas amostra, o que confirma que é possível construir um modelo preditivo robusto, com recurso a um número limitado de variáveis, que apresentam uma forte relação espacial com os deslizamentos estudados. 

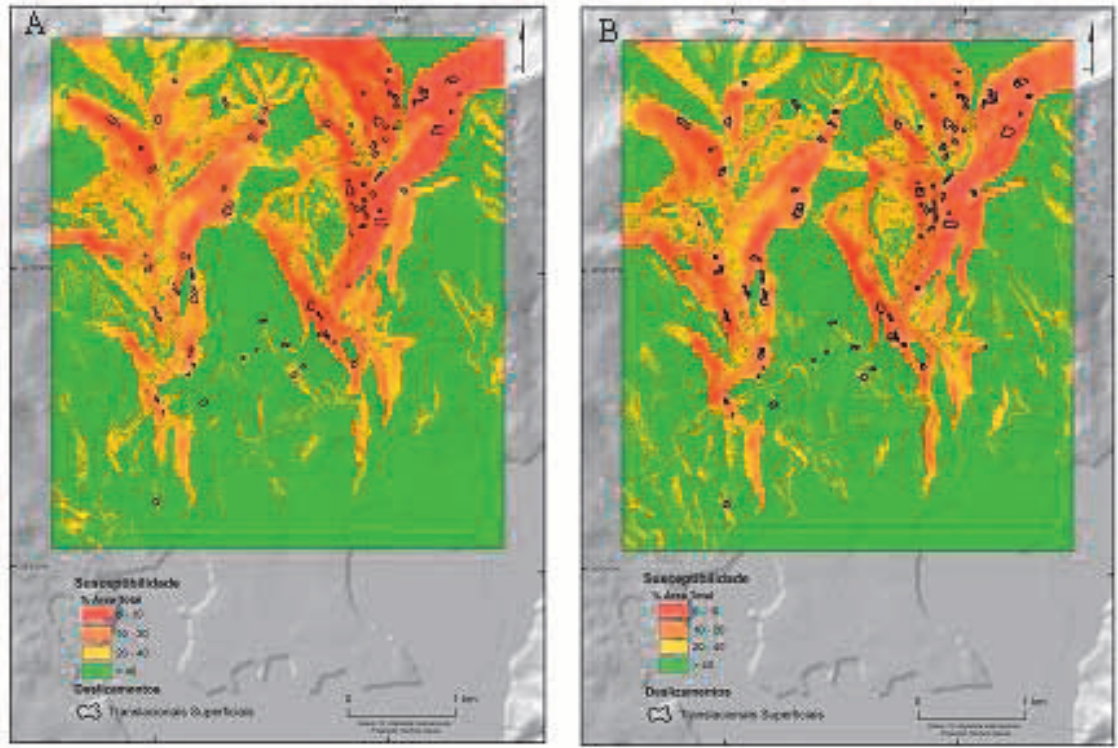

Fig. 5 - Mapas de susceptibilidade a deslizamentos translacionais superficiais na área de Fanhões-Trancão A - Modelação com 7 variáveis; B - Modelação com 3 variáveis.

Fig. 5 - Shallow translational slide susceptibility map of the Fanhões-Trancão test site $A$ - Model with 7 variables; $B$ - Model with 3 variables.
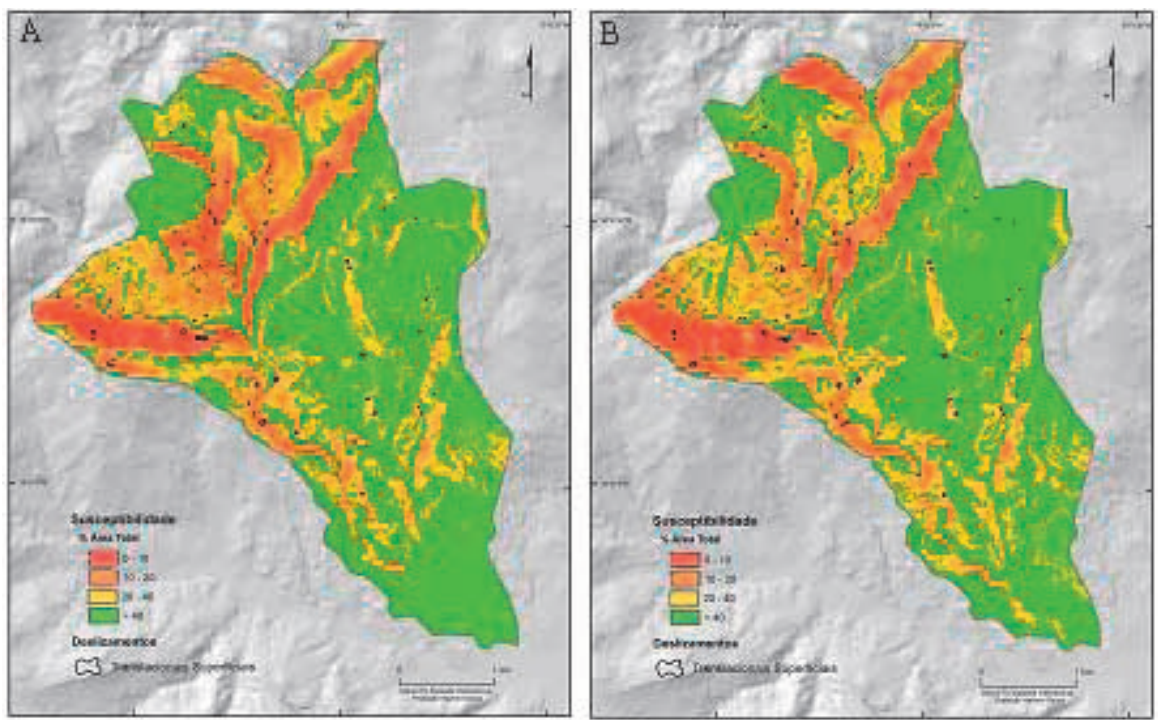

Fig. 6 - Mapas de susceptibilidade a deslizamentos translacionais superficiais na área de Lousa-Loures A - Modelação com 7 variáveis; B - Modelação com 3 variáveis.

Fig. 6-Shallow translational slide susceptibility map of the Lousa-Loures test site $A$-Model with 7 variables; $B$-Model with 3 variables. 


\section{CONCLUSÃO}

A análise sensitiva, aplicada em duas áreas amostra (Fanhões-Trancão e Lousa-Loures) com características geológicas e geomorfológicas similares, foi efectuada com o objectivo de aferir a importância relativa de cada factor de predisposição na distribuição dos movimentos de vertente e, ao mesmo tempo, avaliar a variação na qualidade dos modelos preditivos decorrente do número de variáveis consideradas.

Os resultados obtidos permitiram identificar um mesmo conjunto de três variáveis que se destacam pela associação espacial aos deslizamentos translacionais superficiais nas duas áreas estudadas: declive, unidades geomorfológicas e exposição das vertentes. O facto de as variáveis com melhor capacidade preditiva se repetirem nas duas áreas amostra reforça a ideia de que é possível a exportação de modelos de susceptibilidade entre áreas semelhantes do ponto de vista geológico e geomorfológico, sempre que seja viável a validação dos modelos, pela disponibilidade dos inventários de movimentos de vertente para ambas as áreas (Piedade, 2009; Piedade et al., 2010b).

A análise sensitiva mostrou que a modelação da susceptibilidade com 2, 3, 4, 5,6 ou 7 variáveis não produz diferenças muito significativas ao nível dos resultados preditivos dos modelos, tendo ficado demonstrado que não existe relação linear entre o número de variáveis presentes no modelo e a respectiva capacidade preditiva. Pese embora a inclusão de variáveis adicionais ter incrementado, ainda que ligeiramente, a qualidade preditiva dos modelos na área de Fanhões-Trancão, o mesmo não se verificou na área de Lousa-Loures, pelo que é lícito concluir que o incremento de mais variáveis não gera, necessariamente, melhores resultados preditivos nos modelos de susceptibilidade.

A produção de mapas de susceptibilidade com recurso a um pequeno grupo de variáveis que têm uma forte relação espacial com os movimentos de vertente revela-se, assim, uma boa solução, tanto mais que esta opção tende a produzir manchas territoriais mais homogéneas, uma vez que existem menos unidades de condição única (resultantes das possíveis combinações das classes das 3 variáveis em questão), por comparação com as obtidas com o cruzamento de mais variáveis. A este respeito, refira-se que a homogeneidade das classes de susceptibilidade é sempre desejável nos trabalhos aplicados ao ordenamento do território, uma vez que facilita a gestão territorial das áreas perigosas.

Apesar do exposto, não é aconselhável eliminar à partida as variáveis que se presuma terem menor capacidade preditiva. É mais prudente utilizar, numa primeira fase, o maior número possível de variáveis que possam ser consideradas como factores de predisposição (i.e., que evidenciem relações causa-efeito com os movimentos de vertente) e, numa fase posterior, simplificar os modelos de predição por análise sensitiva das variáveis, eliminando os factores que pouco ou nada acrescentam à predição. 


\section{AGRADECIMENTOS}

Este trabalho faz parte do projecto "MapRisk - Metodologias de avaliação de perigosidade e risco de movimentos de vertente nos Planos Municipais de Ordenamento do Território" (PTDC/ GEO/68227/2006), financiado pela Fundação para a Ciência e Tecnologia.

Os autores agradecem aos revisores do presente artigo que com as suas críticas e sugestões permitiram aperfeiçoar a qualidade do conteúdo.

\section{BIBLIOGRAFIA}

Carrara A L (1993) Uncertainty in evaluating landslide hazard and risk. In Nemec J, Nigg J M, Siccardi F (Eds.) Predictions and perception of natural hazards. Kluwer Academic Publishers. Dordrecht: 101-109.

Carrara A, Guzzetti F, Cardinali M, Reichenback P (1999) Use of GIS technology in the prediction and monitoring of landslide hazard. Natural Hazards, 20: 117-135.

Chacón J, Irigaray C, Fernández T, Hamdouni R E (2006) Engineering geology maps: landslides and geographical information systems. Engineering Geology, 65: 341-411.

Chung C F, Fabbri A (2005) Systematic procedures of landslide hazard mapping for risk assessment using spatial prediction models. In Glade $\mathrm{T}$, Anderson M G, Crozier M J (Eds.) Landslide Hazard and Risk. Wiley, Chichester: 139-174.

Fabbri A, Chung C F, Napolitano P, Remondo J, Zêzere J L (2002) Prediction rate functions of landslide susceptibility applied in the Iberian Peninsula. In Brebbia C A (ed.) Risk Analysis III. Series: Management Information Systems, vol. 5. WIT Press, Southampton: 703-718.

Ferreira A B (1984) Mouvements de terrain dans la Région au Nord de Lisbonne. Conditions morphostructurales et climatiques. Mouvements de Terrain. Colloque de Caen, Documents du B.R.G.M., 83: 485-494.

Guzzetti F, Cardinali M, Reichenbach P, Carrara A (2000) Comparing landslide maps: a case study in the Upper Tiber River Basin, Central Italy. Environmental Management, 25 (3): 247-263.

Guzzetti F, Carrara A, Cardinali M, Reichenbach P (1999) Landslide hazard evaluation: a review of current techniques and their application in a multi-scale study, Central Italy. Geomorphology, 31: 181-216.
Guzzetti F, Reichenbach P, Ardizzone F, Cardinali M, Galli M (2006) Estimating the quality of landslide susceptibility models. Geomorphology, 81: 166-184.

Instituto Nacional de Engenharia, Tecnologia e Inovação I.P. (2007) Carta Geológica de Portugal. Folha 34-B Loures. Departamento de Geologia, Ministério da Econonia e da Inovação, Lisboa.

Lee S (2004) Application of likelihood ratio and logistic regression models to landslide susceptibility mapping using GIS. Environmental Management, 34: 223-232.

Piedade A (2009) Modelação especial em Sistemas de Informação Geográfica da susceptibilidade a deslizamentos na área de Lousa-Loures. Dissertação de Mestrado. Faculdade de Ciências Sociais e Humanas da Universidade Nova de Lisboa, Lisboa.

Piedade A, Zêzere J L, Garcia R A C, Oliveira S C (2010a) Avaliação e validação de modelos de susceptibilidade a deslizamentos em áreas homogéneas na região a Norte de Lisboa. $16^{\circ}$ Congresso da APDR - Regiões de Charneira, Canais de Fronteira e Nós, Universidade da Madeira, Funchal, p. 1318-1333.

Piedade A, Zêzere J L, Tenedório J A, Garcia R A C, Oliveira S C, Rocha J (2010b) Generalization of landslide susceptibility models in geologic-geomorphologic similar context. Geophysical Research Abstracts, vol. 12. EGU2010-3666-2. $1 \mathrm{p}$.

Remondo J, González A, Terán J R D, Cendredo A, Fabri A, Chung C (2003) Validation of landslide susceptibility maps; exemples and applications from a case study in northern Spain. Natural Hazards, 30: 437-440.

Santacana N, Baeza B, Corominas J, Paz A, Marturiá J (2003) A GIS-based multivariate statistical 
analysis for shallow landslide susceptibility mapping in La Pobla de Lillet Area (Eastern Pyrenees, Spain). Natural Hazards, 30: 281-295 .

Soeters R, Van Westen C J (1996) Slope instability recognition, analysis and zonation. In Turner A K, Schuster R L (Eds.) Landslides, investigation and mitigation. Transportation Research Board, National Research Council, Special Report 247. National Academy Press, Washington D.C., U.S.A.: 129-177.

Van Den Eeckhaut M, Marre A, Joesen J (2010) Comparison of two landslide susceptibility assessments in the Champagne-Andernne region (France). Geomorphology, 115: 141-155.

Van Wester C J, Castellanos E, Kuriakose S L (2008) Spatial data for landslide susceptibility, hazard, and vulnerability assessment: an overview. Engineering Geology, 102: 112-131.

Varnes D J (1984) Landslide hazard zonation: a review of principles and practice. UNESCO, Paris.

Yan T Z (1988) Recent advances of quantitative prognoses of landslide in China. In Bonnard C (ed.) Landslides. Proceedings of the Fifth International Symposium on Landslides, Lausanne, 2, Balkema, Rotterdam: 1263-1268.

Yin K L, Yan T Z (1988) Statistical prediction models for slope instability of metamorphosed rocks. In Bonnard C (ed.) Landslides. Proceedings of the Fifth International Symposium on Landslides, 2, Balkema, Rotterdam: 1269-1272.

Zêzere J L (2007) Predição probabilística de movimentos de vertente na escala regional. Actes de les Jornades sobre Terrasses i Prevenció de Riscos Naturals. Departement de Medi Ambient, Consell de Mallorca: 17-30.

Zêzere J L (1997) Movimentos de vertente e perigosidade geomorfológica na Região a Norte de
Lisboa. Dissertação de Doutoramento. Faculdade de Letras da Universidade de Lisboa, Lisboa.

Zêzere J L, Ferreira A B, Rodrigues M L (1999) The role of conditioning and triggering factors in the occurrence of landslides: a case study in the area north of Lisbon (Portugal). Geomorphology, 30 (1-2): 133-146.

Zêzere J L, Reis E, Garcia R, Oliveira S, Rodrigues M L, Vieira G, Ferreira A B (2004) Integration of spatial and temporal data for the definition of different landslide hazard scenarios in the area north of Lisbon (Portugal). $\mathrm{Na}$ tural Hazards and Earth System Sciences, 4: 133-146.

Zêzere J L, Garcia R A C, Oliveira S C, Reis E (2005a) Análise sensitiva na avaliação da susceptibilidade a deslizamentos na Região a Norte de Lisboa. X Colóquio Ibérico de Geografia "A Geografia Ibérica no contexto europeu”, Évora.

Zêzere J L, Trigo R, Trigo I (2005b) Shallow and deep landslides induced by rainfall in the Lisbon region (Portugal): assessment of relationships with the North Atlantic Oscillation. Natural Hazards and Earth System Sciences, 5: 331-344.

Zêzere J L, Oliveira S C, Garcia R A C, Reis E (2008) Weighting predisposing factors for shallow slides susceptibility assessment on at the region scale. In Chen Z, Zhang J, Li Z, Wu F, Ho K (eds.) Landslides and Engineered Slopes: from the past to the future. $2{ }^{\circ}$ Volume, London: 1831-1837.

Zbyszewski G (1964) Notícia explicativa da Folha 2 - Loures. Carta Geológica dos Arredores de Lisboa. Escala de 1: 50 000. Direcção-geral de minas e serviços geológicos. Serviços Geológicos de Portugal. Lisboa. 